\title{
Study of Spin-Orbit Coupling Effect on Bismuth (111) Bilayer
}

\author{
M. BienieK*, T. WoŹniak And P. Potasz \\ Department of Theoretical Physics, Faculty of Fundamental Problems of Technology, \\ Wrocław University of Technology, Wybrzeże Wyspiańskiego 27, PL-50-370 Wrocław, Poland
}

\begin{abstract}
Band inversion process in $\mathrm{Bi}(111)$ bilayer is studied using many-orbital tight-binding model, supported by the density-functional theory calculations, with a controllable spin-orbit coupling constant in tight-binding model This effect is important in order to verify a topological nature of this material. We show that after closing of the energy gap leading to crossing of the valence and conduction bands, the second band inversion occurs within a valence band. We analyze orbital composition and spin textures of bands within different regimes. Around a $\Gamma$ point, all spins align in one direction before the first band inversion. Moreover, a change of signs for some spin components after a band inversion is noticed. After the second band inversion, a significant change of orbital contribution of the top of the valence band is observed.
\end{abstract}

DOI: 10.12693/APhysPolA.130.609

PACS/topics: 73.22.Dj, 74.20.Pq

\section{Introduction}

Topological insulators (TI) [1-3] are new state of electronic matter characterized by the so-called $Z_{2}$ invariant [4]. At the boundary between trivial insulator and TI a pair of chiral edge states is predicted to occur, which exhibits unique transport properties, most prominently an absence of backscattering and spin-momentum locking [5]. Due to similarity of the topological insulator phase in two dimensions to integer quantum Hall effect [6] (IQHE), it is called quantum spin Hall effect (QSHE), although no external magnetic field is needed.

Several materials were proposed to be 2D topological insulators including $\mathrm{CdTe} / \mathrm{HgTe}$ [7], InAs/GaSb [8] quantum wells or bismuth (111) bilayers $(\operatorname{Bi}(111))[9]$. Shortly after their theoretical prediction, both quantum well systems were shown experimentally $[10,11]$ to exhibit quantized conductance characteristic of TI. However, it is still subjected to ongoing debate if the transport measurements prove undoubtedly topological nature of this peculiar phase. One of the problems is that in $\mathrm{CdTe} / \mathrm{HgTe}$ quantum wells edge states penetration is relatively large in comparison with e.g. bismuth $(\approx 6 \mathrm{~nm}$ versus $\approx 50 \mathrm{~nm}$ in $\mathrm{CdTe} / \mathrm{HgTe}$ [12]), which can lead to scattering of electrons between edges in nanoribbon geometries, violating quantization of a conductance.

Recently, an experimental observation of the edge states in $\mathrm{Bi}(111)$ on $\mathrm{Bi}_{2} \mathrm{Te}_{3}$ has been reported by Yang et al. [13] and followed by the transport study by Sabater et al. [14]. Additionally, a topological nature of onedimensional edge states in bismuth on clean Bi crystal was shown in scanning tunneling microscope (STM) experiment by Drozdov et al. [15], although an interpretation of these results have been questioned [16]. More generally, the problem of the edge state survival and

\footnotetext{
*corresponding author; e-mail: maciej.bieniek@pwr.edu.pl
}

their topological character in $\mathrm{Bi}(111)$ bilayer on various substrates and for different numbers of layers is still open [17-19].

An existence of edge states in topological insulators is strictly related to the bulk properties of the crystal [4]. A characteristic feature of a transition between trivial and TI phase is a band inversion [7]. This can be controlled by a number of external factors e.g. strain in the system, controlled quantum well width in growth process, or doping $[7,20]$.

In the following work, we investigate the band inversion process in $\mathrm{Bi}(111)$ bilayer controlled by the spinorbit coupling constant. In our studies we use manyorbital tight-binding (TB) model $[9,21]$ whose validity was confirmed by other authors $[14,15]$ and our densityfunctional theory (DFT) calculations. Within DFT results we examine electronic structure and orbital compositions in the whole Brillouin zone. Next, we focus on an effect of the spin-orbit coupling strength on electronic band structure. We show that two band inversions occur, the first between conduction and valence bands and the second between bands within a valence band. We analyze a nature of different regimes by looking at orbital composition of energy bands and spin textures.

\section{Methods}

The $a b$ initio calculations were conducted with ABINIT software [22], which implements DFT formalism. The slabs of $\mathrm{Bi}$ bilayers were separated by vacuum region of $10 \AA$. Atoms were modelled in the frame of fully relativistic projector augmented wave (PAW) atomic datasets [23] within the general gradient approximations (GGA) of the exchange-correlation functional [24]. Structural parameters were optimized until the interatomic forces were smaller than $10^{-7} \mathrm{Ha} / \mathrm{bohr}$. A $8 \times 8 \times 1$ and $16 \times 16 \times 1$ Monkhorst-Pack $k$-point grids were used for structural optimization and density of states calculations, respectively. The plane wave basis cutoff was set to $20 \mathrm{Ha}$. 
Bi bilayer was modelled in a hexagonal unit cell with a two-atom basis. The optimized lattice constant $a=$ $4.6757 \AA$ and bilayer thickness $h=1.6072 \AA$ are in good agreement with experimental values [25]. The band structure was calculated along a path between highsymmetry points in the first Brillouin zone: $M-\Gamma-K-$ $M$, whose coordinates are $M=(1 / 2,0), \Gamma=(0,0)$, $K=(1 / 3,1 / 3)$.

For calculations within four orbital tight-binding model, we use a parametrization developed by Liu and Allen [21] with modification proposed by Murakami [9], in which all interlayer interactions are set to 0 . To study the effect of spin-orbit interaction, the original coupling parameter $\lambda=1.5 \mathrm{eV}$ (from Ref. [21]) is varied between 0 and $2 \mathrm{eV}$. In order to analyze spin textures we look at the expectation values of spin operators $\left\langle\psi_{\text {val(cond) }}\left|\hat{\sigma}_{x, y, z}\right| \psi_{\text {val(cond) })}\right\rangle$ in an appropriate valence (conduction) band. In our calculations, a Zeeman spin splitting of $50 \mathrm{meV}$ is introduced to remove Kramers degeneracy of bands. This effect can be realized experimentally by applying an in-plane magnetic field.

\section{Results}

The electronic band structure and projected density of states of $\mathrm{Bi}(111)$ bilayer calculated with the DFT method are shown in Fig. 1 in the left and right parts, respectively. $\mathrm{Bi}(111)$ bilayer is an indirect gap semiconductor with the energy gap of $E_{\text {gap }}=0.45 \mathrm{eV}$. The conduction band minimum is located at the $\Gamma$ point and it consists mainly of hybridized $p_{x}$ and $p_{y}$ orbitals. In the valence band we observe two maxima near the $\Gamma$ point. All $p$ orbitals have equal contribution to the electronic structure in this region.

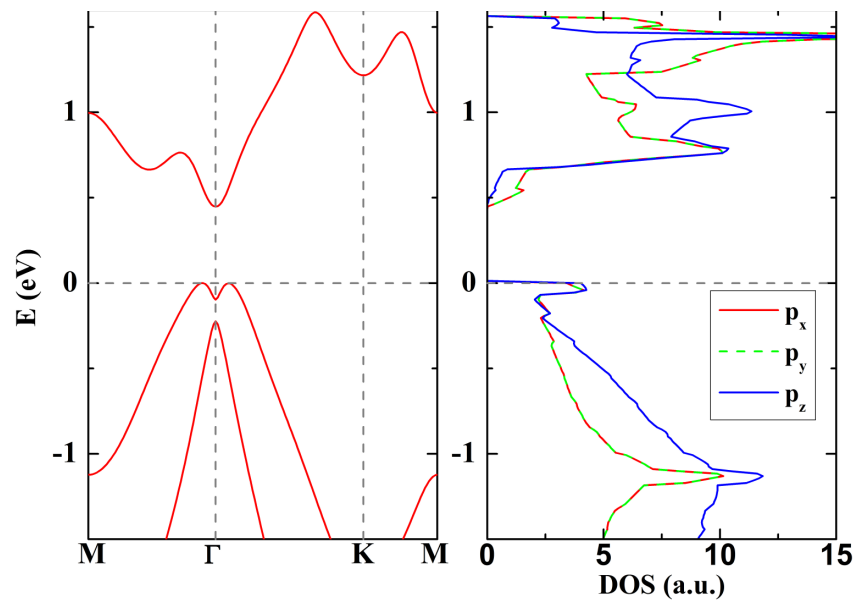

Fig. 1. A band structure (left) along $M-\Gamma-K-M$ line and projected density of states (right) of $\mathrm{Bi}(111)$ bilayer from DFT calculations with the spin-orbit coupling.

In Fig. 2 detailed tight-binding model study of an evolution of the electronic structure and the orbital contributions with respect to the spin-orbit coupling (SOC)
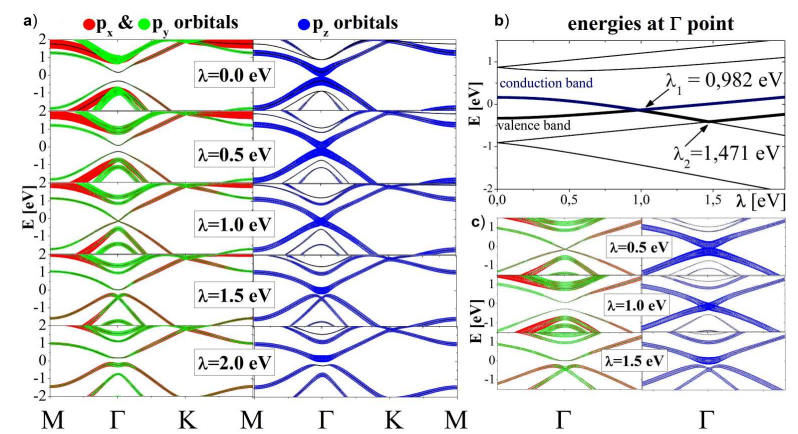

Fig. 2. (a) Evolution of the electronic structure along $M-\Gamma-K-M$ line for different values of the spin-orbit coupling parameter $\lambda$ ranging from $\lambda=0 \mathrm{eV}$ to $\lambda=$ $2 \mathrm{eV}$. Red, green and blue circles denote $p_{x}, p_{y}$, and $p_{z}$ orbitals, respectively. Circle radius denotes relative orbital contribution extracted from eigenfunction analysis. (b) Evolution of the energies at the $\Gamma$ point as a function of the spin-orbit coupling strength $\lambda$. Fermi level is set to $E_{\mathrm{F}}=0 \mathrm{eV}$. Two band inversions are observed, the first between conduction and valence bands (located at $\lambda_{1}=0.982 \mathrm{eV}$ ) and the second between two valence bands (at $\lambda_{2}=1.471 \mathrm{eV}$ ). (c) Close up at the $\Gamma$ point and around the energy gap region before the first inversion $(\lambda=0.5 \mathrm{eV})$, after $(\lambda=1.0 \mathrm{eV})$ and after the second inversion $(\lambda=1.5 \mathrm{eV})$. All symbols are as in (a). Conduction band retains $p_{z}$ character, where the valence band changes from $p_{z}$ to $p_{x}-p_{y}$ mixture. Slight asymmetry results from a choice of directions at the Brillouin zone: left from $\Gamma$ to $M$ and right from $\Gamma$ to $K$.

parameter $\lambda$ is presented. For $\lambda=0.0 \mathrm{eV}$ band edges consist mostly of $p_{z}$ orbitals (see Fig. 2a). With the increase of SOC, the energy gap closes and reopens, see Fig. 2b, which is characteristic of topological phase transition [7,26]. A critical point is observed for $\lambda_{1}=$ $0.982 \mathrm{eV}$ (Fig. 2b). An increase of $\lambda$ above $\lambda_{1}=0.982 \mathrm{eV}$ results in opening slightly an indirect gap, contrary to a direct gap for smaller values of the SOC parameter. Up to $\lambda_{2}=1.471 \mathrm{eV}$, a valence band retains $p_{z}$ character. At $\lambda_{2}=1.471 \mathrm{eV}$ two valence bands with different orbital compositions cross each other. A further increase of SOC leads to an inversion within the valence band. After this, a top of the valence band changes its character from $p_{z}$ orbitals to a mixture of $p_{x}$ and $p_{y}$ orbitals, which is visible in Fig. 2c for $\lambda=1.5 \mathrm{eV}$. We note that an orbital character of the conduction band in our TB model (mainly $p_{z}$ contribution) for an experimentally expected value of SOC, $\lambda \approx 1.5 \mathrm{eV}$, slightly differs from our DFT results, where a bottom of the conduction band is mostly composed of $p_{x}$ and $p_{y}$ orbitals. However, we note that a shape of the energy bands remains in a good qualitative agreement between both calculation methods.

In order to study spin textures of the valence and conduction bands, we lift a Kramers degeneracy by introducing the Zeeman spin splitting term with a value $50 \mathrm{meV}$, which is shown in the inset of Fig. 3 as a function of the SOC. The largest splitting is observed when 


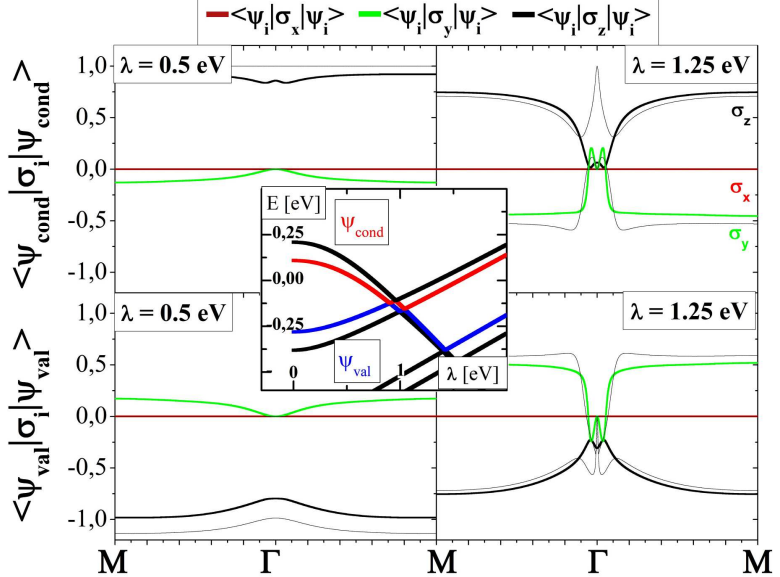

Fig. 3. Spin texture of Zeeman-splitted valence $\left(\left\langle\psi_{\text {val }}\left|\hat{\sigma}_{i}\right| \psi_{\text {val }}\right\rangle\right)$ and conduction $\left(\left\langle\psi_{\text {cond }}\left|\hat{\sigma}_{i}\right| \psi_{\text {cond }}\right\rangle\right)$ bands for two SOC parameters $(\lambda=0.5 \mathrm{eV}$ and $\lambda=1.25 \mathrm{eV}$ ) on line $M-\Gamma-M$ in the Brillouin zone. A value of the Zeeman splitting is taken to be equal to $50 \mathrm{meV}$. Red, green and black lines represent expected values of the Pauli matrices $\hat{\sigma}_{x}, \hat{\sigma}_{y}, \hat{\sigma}_{z}$, respectively, in state $\psi_{\text {val }}$ and $\psi_{\text {cond }}$ (valence and conduction band eigenstates). Black thin lines show corresponding spin textures for $\lambda=0.0 \mathrm{eV}$ (left part) and $\lambda=1.5 \mathrm{eV}$ (right part). Inset: Energy as a function of the SOC parameter $\lambda$ for Zeeman-splitted bands at the $\Gamma$ point. A red (blue) line corresponds to a conduction (valence) band with $\psi_{\text {cond }}\left(\psi_{\text {val }}\right)$ eigenfunction.

SOC is turned off, and decreases with an increase of the SOC strength. In Fig. 3, spin textures of the valence and conduction bands are presented for $\lambda=0.5 \mathrm{eV}$ and $\lambda=1.25 \mathrm{eV}$. These values of SOC corresponds to two situations: before the energy gap closes and after it reopens. One can observe a vanishing of $\left\langle\hat{\sigma}_{x}\right\rangle$ component in all cases and variation of $\left\langle\hat{\sigma}_{z}\right\rangle$ and $\left\langle\hat{\sigma}_{y}\right\rangle$ components, which corresponds to spin rotation in $y-z$ plane. A $\left\langle\hat{\sigma}_{z}\right\rangle$ sign for the conduction (valence) band is positive (negative) in both regimes. In a case of $\lambda=0.5 \mathrm{eV}$, an expectation value of $\left\langle\hat{\sigma}_{y}\right\rangle$ does not change a sign along $M-\Gamma-M$ line, for both conduction and valence bands. This corresponds to an alignment of spins approximately in one direction. On the other hand, after the band inversion (for $\lambda=1.25 \mathrm{eV}$ ), some spin texture is observed. At the $\Gamma$ point $\left\langle\hat{\sigma}_{y}\right\rangle$ goes exactly to 0 . Moving away from the $\Gamma$ point, for the conduction band, $\left\langle\hat{\sigma}_{y}\right\rangle$ first increases up to some maximal value, after which again drops to zero. Going further from the $\Gamma$ point, $\left\langle\hat{\sigma}_{y}\right\rangle$ becomes again nonzero, increasing but in negative values. Inverse situation is observed for the valence band, see lower right part in Fig. 3.

\section{Conclusions}

We investigated a band structure of $\mathrm{Bi}(111)$ bilayer under the band inversion transition caused by changing of the SOC strength. We noticed two band crossings, the first closing the energy gap and inverting the conduction and valence bands, and the second between two bands within a valence band. We show a variation of an orbital composition of the conduction and valence bands in different regimes, and an appearance of the spin texture after a conduction-valence band inversion.

\section{Acknowledgments}

P.P. acknowledges partial financial support from National Science Center (NCN), Poland, grant Sonata no. 2013/11/D/ST3/02703. M.B. thanks Foundation for Polish Science (FNP) Master grant no. 10/2012. DFT calculations were performed in Wrocław Center for Networking and Supercomputing.

\section{References}

[1] J. Moore, Nature 464, 194 (2010).

[2] M.Z. Hasan, C.L. Kane, Rev. Mod. Phys. 82, 3045 (2010).

[3] X.-L. Qi, S.-C. Zhang, Rev. Mod. Phys. 83, 1057 (2011).

[4] C.L. Kane, E.J. Mele, Phys. Rev. Lett. 95, 146802 (2005).

[5] C.L. Kane, E.J. Mele, Phys. Rev. Lett. 95, 226801 (2005).

[6] K. von Klitzing, G. Dorda, M. Pepper, Phys. Rev. Lett. 45, 494 (1980).

[7] B.A. Bernevig, T.L. Hughes, S.-C. Zhang, Science 314, 1757 (2006).

[8] C. Liu, T.L. Hughes, X.-L. Qi, K. Wang, S.-C. Zhang, Phys. Rev. Lett. 100, 236601 (2008).

[9] S. Murakami, Phys. Rev. Lett. 97, 236805 (2006).

[10] M. König, S. Wiedmann, C. Brüne, A. Roth, H. Buhmann, L. Molenkamp, X.-L. Qi, S.-C. Zhang, Science 318, 766 (2007).

[11] I. Knez, R.-R. Du, G. Sullivan, Phys. Rev. Lett. 107, 136603 (2011).

[12] M. Wada, S. Murakami, F. Freimuth, G. Bihlmayer, Phys. Rev. B 83, 121310(R) (2011).

[13] F. Yang, L. Miao, Z.F. Wang, M.-Y. Yao, F. Zhu, Y.R. Song, M.-X. Wang, J.-P. Xu, A.V. Fedorov, Z. Sun, G.B. Zhang, Canhua Liu, Feng Liu, Dong Qian, C.L. Gao, Jin-Feng Jia, Phys. Rev. Lett. 109, 016801 (2012).

[14] C. Sabater, D. Gosalbez-Martinez, J. FernandezRossier, J.G. Rodrigo, C. Untiedt, J.J. Palacios, Phys. Rev. Lett. 110, 176802 (2013).

[15] I.K. Drozdov, A. Alexandradinata, S. Jeon, S. NadjPerge, H. Ji, R.J. Cava, B.A. Bernevig, A. Yazdani, Nat. Phys. 10, 664 (2014).

[16] H.W. Yeom, K.-H. Jin, S.-H. Jhi, arXiv:1511.02330v1.

[17] Z.-Q. Huang, F.-C. Chuang, C.-H. Hsu, Y.-T. Liu, H.-R. Chang, H. Lin, A. Bansil, Phys. Rev. B 88, 165301 (2013).

[18] Z. Liu, C.-X. Liu, Y.-S. Wu, W.-H. Duan, F. Liu, J. Wu, Phys. Rev. Lett. 107, 136805 (2011). 
[19] L. Chen, Z.F. Wang, F. Liu, Phys. Rev. B 87, 235420 (2013).

[20] C.L. Kane, Phys. Rev. B 76, 045302 (2007).

[21] R.E. Allen, Phys. Rev. B 52, 1566 (1995).

[22] X. Gonze, B. Amadon, P. Anglade, J. Beuken, F. Bottin, P. Boulanger, F. Bruneval, D. Caliste, R. Caracas, M. Cote, T. Deutsch, L. Genovese, Ph. Ghosez, M. Giantomassi, S. Goedecker, D.R. Hamann, P. Hermet, F. Jollet, G. Jomard, S. Leroux, M. Mancini, S. Mazevet, M.J.T. Oliveira, G. Onida, Y. Pouillon, T. Rangel, G.-M. Rignanese, D. Sangalli, R. Shaltaf, M. Torrent, M.J. Verstraete, G. Zerah, J.W. Zwanziger, Comput. Phys. Commun. 180, 2582 (2009).
[23] N. Holzwarth, A. Tackett, G. Matthews, Comput. Phys. Commun. 135, 329 (2001).

[24] J. Perdew, K. Burke, M. Ernzerhof, Phys. Rev. Lett. 77, 3865 (1996).

[25] P. Hoffman, Prog. Surf. Sci. 81, 191 (2006).

[26] P. Dziawa, B.J. Kowalski, K. Dybko, R. Buczko, A. Szczerbakow, M. Szot, E. Łusakowska, T. Balasubramanian, B.M. Wojek, M.H. Berntsen, O. Tjernberg, T. Story, Nat. Mater. 11, 1023 (2012). 